\title{
Flutter and parametric stability analysis of axially moving composite graphene sheets
}

\author{
A. Azrar ${ }^{1}$, N. Fakri' ${ }^{1}$, A. A. Aljinaidi ${ }^{2}$ and L. Azrar ${ }^{2,3}$ \\ ${ }^{1}$ Mathematical Modeling and Control, Faculty of Sciences and Techniques of Tangier \\ University Abdelmalek Essaadi, BP 416, Tangier, Morocco, \\ ${ }^{2}$ Departement of Mechanical Engineering, Faculty of Engineering, Kind Abdulaziz \\ University, Jeddah, Saudi Arabia \\ ${ }^{3}$ Research Center STIS, Team M2CS, ENSET, Mohammed V University, Rabat, Morocco \\ abdellhadiazrar@yahoo.fr; nfakri@yahoo.fr; aljinaidi@kau.edu.sa; 1.azrar@um5s.net.ma,
}

\begin{abstract}
The dynamic analysis instability of axially moving rectangular composite graphene sheets with visco elastic foundation is modeled and numerically simulated for various boundary conditions based on the differential quadrature method (DQM). The partial differential equation of motion based on the nonlocal elasticity and the Kirchhoff plate theories is given. The Galerkin and harmonic balance methods are used for the linear and parametric vibration analysis. The influences of nonlocal parameter, the fibers orientation and the viscoelastic foundation effects on the dynamic behaviors of the rectangular graphene sheet as well as the instabilities induced by the time dependent axial speed and its excitation frequency are investigated.
\end{abstract}

\section{Keywords}

Dynamic analysis, Instability, Composite graphene sheets, Differential quadrature, Nonlocal elasticity.

\section{Introduction}

Vibration analysis of structures traversed by moving load is a well-known subject in structural mechanics and has been of great interest in many engineering disciplines and studied by many researchers. Several researches for the description of dynamic responses of the isotropic beams and plates due to the moving loads and masses based on the various methods were developed. A recent study by Nami and Janghorban [1] proposed the dynamic analysis of isotropic nanoplates subjected to moving load using state-space method based on nonlocal second order plate theory. Vibration response of CNTs is considered by many investigators using local and nonlocal theories. Analytical and numerical modeling of higher order free vibration characteristics of single walled Carbon NanoTubes and CNT conveying fluid have been elaborated by Azrar et al [2-3]. In this paper, the problem of composite nanoplates traversed by a time dependent moving axially load is studied and the dynamic behavior of the system components is investigated.
A numerical procedure based on the differential quadrature method and multimodal formulations has been elaborated for the linear dynamic and parametric instabilities of axially moving load. Frequency and time domains are considered and instability analyses have been performed with respect to the considered influencing parameters based numerical procedure.

\section{Mathematical formulation}

Based on the nonlocal and Kirchhoff plate theories, the partial dimensionless differential equation of motion for axially moving composite nanoplates with visco-elastic foundation is given by:

$$
\begin{aligned}
& \left(1+\left(K_{G}-V^{2}\right) \mu^{2}\right) \frac{\partial^{4} w}{\partial \xi^{4}}+\left(\mu^{2} K_{G}+\eta_{22}\right) \lambda^{4} \frac{\partial^{4} w}{\partial \zeta^{4}} \\
& +\left(\left(2 K_{G}-V^{2}\right) \mu^{2}+2 \eta_{12}+4 \eta_{66}\right)+\lambda^{2} \frac{\partial^{4} w}{\partial \zeta^{2} \partial \xi^{2}} \\
& -\left(K_{G}+\mu^{2} K_{E}-V^{2}\right) \frac{\partial^{2} w}{\partial \xi^{2}}+4 \eta_{16} \lambda \frac{\partial^{4} w}{\partial \xi^{3} \partial \zeta}-2 \mu^{2} V \frac{\partial^{4} w}{\partial \xi^{3} \partial \tau} \\
& -\left(\mu^{2} K_{E}+K_{G}\right) \lambda^{2} \frac{\partial^{2} w}{\partial \zeta^{2}}+4 \eta_{26} \lambda^{3} \frac{\partial^{4} w}{\partial \xi \partial \zeta^{3}}+2 V \frac{\partial^{2} w}{\partial \xi \partial \tau} \\
& +\frac{\partial^{2} w}{\partial \tau^{2}}+K_{E} w+C_{E}\left(\frac{\partial w}{\partial \tau}-\mu^{2}\left(\frac{\partial^{3} w}{\partial \xi^{2} \partial \tau}-\lambda^{2} \frac{\partial^{3} w}{\partial \zeta^{2} \partial \tau}\right)\right) \\
& -\mu^{2}\left(\frac{\partial^{4} w}{\partial \xi^{2} \partial \tau^{2}}+2 V \lambda^{2} \frac{\partial^{4} w}{\partial \xi \partial \zeta^{2} \partial \tau}+\lambda^{2} \frac{\partial^{4} w}{\partial \zeta^{2} \partial \tau^{2}}\right)=0(1)
\end{aligned}
$$

Where w, $\mathrm{K}_{E}, K_{G}$ and $C_{E}$ are the dimensionless transvers deflection, Winkler foundation modulus, the shear layer foundation stiffness of Pasternak model, and the damping coefficient of the foundation respectively. $\mu$ is the nonlocal parameter, $\mathrm{V}$ is the dimensionless axial speed. $\lambda=a / b$ in which $a$ and $b$ are respectively the length and width of the graphene sheet.

The transformed reduced stiffness constants $\eta_{12}, \eta_{22}, \eta_{16}$ and $\eta_{66}$ are given by: 
$\eta_{12}=\frac{D_{12}}{D_{11}} ; \eta_{16}=\frac{D_{16}}{D_{11}} ; \eta_{22}=\frac{D_{22}}{D_{11}} ; \eta_{26}=\frac{D_{26}}{D_{11}} ; \eta_{66}=\frac{D_{66}}{D_{11}}$

where $D_{11}=\frac{h^{3}}{12}\left(Q_{11} c^{4}+2\left(Q_{12}+2 Q_{66}\right) c^{2} s^{2}+Q_{22} s^{4}\right)$;

$D_{12}=\frac{h^{3}}{12}\left(\left(Q_{11}+Q_{12}-4 Q_{66}\right) c^{2} s^{2}+Q_{12}\left(c^{4}+s^{4}\right)\right) ;$

$D_{22}=\frac{h^{3}}{12}\left(Q_{11} s^{4}+2\left(Q_{12}+2 Q_{66}\right) c^{2} s^{2}+Q_{22} c^{4}\right) ;$

$D_{16}=\frac{h^{3}}{12}\left(\left(Q_{11}-Q_{12}-2 Q_{66}\right) c^{3} s+\left(Q_{12}-Q_{22}+2 Q_{66}\right) c s^{3}\right) ;$

$D_{26}=\frac{h^{3}}{12}\left(\left(Q_{11}-Q_{12}-2 Q_{66}\right) c s^{3}+\left(Q_{12}-Q_{22}+2 Q_{66}\right) c^{3} s\right) ;$

$D_{66}=\frac{h^{3}}{12}\left(\left(Q_{11}+Q_{22}-2 Q_{12}-2 Q_{66}\right) c^{2} s^{2}+Q_{66}\left(c^{4}+s^{4}\right)\right) ;$

where $c=\cos (\theta) ; s=\sin (\theta)$ and $\theta$ is the angle between the fiber direction and the longitudinal axis of the plate. The reduced stiffness constants $Q_{11}, Q_{22}, Q_{12}$ and $Q_{66}$ are given by

$Q_{11}=\frac{E_{1}}{1-v_{12} v_{21}} ; Q_{22}=\frac{E_{2}}{1-v_{12} v_{21}} ; Q_{12}=\frac{v_{12} E_{2}}{1-v_{12} v_{21}} ; Q_{66}=G_{12}$

where $E_{1}, E_{2}, E_{12}$ and $v_{12}$ are the mechanical elastic constants of rectangular plate and the coefficient $v_{21}$ can be determined according to the following relation $E_{1} / v_{12}=E_{2} / v_{21}$

Various boundary conditions are considered.

Based on the differential quadrature method in the space domain, Equation (1) can be rewritten in the following matrix form:

$$
[M]\{\ddot{w}\}+[C]\{\dot{w}\}+[K]\{w\}=0
$$

where the matrices $\mathrm{M}, \mathrm{C}$ and $\mathrm{K}$ are given by:

$$
\begin{aligned}
& M=I_{1}-\mu^{2}\left(H_{x}^{2}+\lambda^{2} H_{y}^{2}\right) ; \\
& C=\left(2 H_{x}^{1}-2 \mu^{2}\left(H_{x}^{3}+\lambda^{2} H_{x, y}^{(1,2)}\right)\right) V \\
& +C_{E}\left(I_{1}-\mu^{2}\left(H_{x}^{2}+\lambda^{2} H_{y}^{2}\right)\right) ; \\
& K=\left(V^{2}-K_{G}-\mu^{2} K_{E}\right) H_{x}^{2}-\left(K_{G}+\mu^{2} K_{E}\right) \lambda^{2} H_{y}^{2} \\
& +\left(1+\left(K_{G}-V^{2}\right) \mu^{2}\right) H_{x}^{4}+\left(\eta_{22}+\mu^{2} K_{G}\right) \lambda^{4} H_{y}^{4} \\
& +\left(2 \eta_{12}+4 \eta_{66}-\mu^{2}\left(V^{2}-2 K_{G}\right)\right) \lambda^{2} H_{x, y}^{(2,2)} \\
& +4 \lambda^{3} \eta_{26} H_{x, y}^{(1,3)}+4 \lambda \eta_{16} H_{x, y}^{(3,1)} ; w=\left[w_{1}, w_{2}, \ldots, w_{n}\right]^{T}
\end{aligned}
$$

The expressions of $H_{i, j}^{k, l}$ are omitted here due to limited space.

\section{Flutter and divergence formulations}

For the analysis of complex eigenmodes and associated complex eigenfrequencies, the problem (2) is reduced to the following speed dependent eigenvalue problem:

$\int \Gamma Y=\omega Y$,

$\left\{\Gamma=\left(\begin{array}{cc}M & 0 \\ 0 & M\end{array}\right)^{-1}\left(\begin{array}{ll}0 & K \\ K & C\end{array}\right), Y=\left\{\begin{array}{l}W \\ \omega W\end{array}\right\}\right.$,

where

$$
K_{h}=\left[K_{S}\right]-\left[K_{D}\right]\left[K_{B}\right]^{-1}\left[K_{C}\right]
$$

For dynamic analysis, the frequency and time domains are considered and the focus is first on the frequency domain.

\section{Dynamic stability analysis}

In this paper, a pulsating force is considered and the axially speed is assumed to be harmonically fluctuating and has the following dimensionless form:

$V(\tau)=V_{0}(1+\eta \cos (\Omega \tau))$

where $\mathrm{V}_{0}$ is the mean of dimensionless axially speed, $\eta$ the amplitude of the harmonic fluctuation and $\Omega$ its frequency. The deflection of the nanoplates can be approximated by the first $\bar{N}$ modes using the Galerkin procedure.

$w(\xi, \zeta, \tau)=\sum_{i=1}^{\bar{N}} \varphi_{i}(\xi, \zeta) q_{i}(\tau)$

where $\varphi_{i}(\xi, \zeta)$ and $q_{i}(t)$ are the numerical eigenmodes and the unknown functions of time. Substituting Eqs. (4), (5) into (2), and according to the orthogonal property of the eigenmodes, the following differential equation is obtained:

$$
\begin{aligned}
& M \ddot{q}_{i}(\tau)+\left[C_{0}+C_{1} \cos (\Omega \tau)\right] \dot{q}_{i}(\tau)+ \\
& {\left[K_{0}+K_{1} \cos (\Omega \tau)+K_{2} \cos ^{2}(\Omega \tau)\right] q_{i}(\tau)=0}
\end{aligned}
$$

where the matrices $\mathrm{C}_{1}, \mathrm{~K}_{1}$ and $\mathrm{K}_{2}$ are given by:

$C_{1}=\left(2 H_{x}^{1}-2 \mu^{2}\left(H_{x}^{3}+\lambda^{2} H_{x, y}^{(1,2)}\right)\right) V_{0}$;

$K_{1}=2\left(H_{x}^{2}-\mu^{2}\left(H_{x}^{4}+\lambda^{2} H_{x, y}^{(2,2)}\right)\right) V_{0}^{2}$;

$K_{2}=\left(H_{x}^{2}-\mu^{2}\left(H_{x}^{4}+\lambda^{2} H_{x, y}^{(2,2)}\right)\right) V_{0}^{2}$;

\section{Parametric instability formulation}

The generalized coordinate $\mathrm{q}_{\mathrm{j}}(\tau)$ is assumed periodic and is expressed in the following form [4]:

$q_{j}(\tau)=\sum_{k=0}^{\bar{M}}\left\{a_{k} \sin \left(\frac{(2 k+1)}{2} \Omega \tau\right)+b_{k} \cos \left(\frac{(2 k+1)}{2} \Omega \tau\right)\right\}$

Based on the two-mode and one-harmonic approaches the instability boundaries can be obtained by solving the following nonlinear algebraic equation.

$$
\left(\frac{\Omega}{2}\right)^{8} \beta_{8}+\left(\frac{\Omega}{2}\right)^{6} \beta_{6}+\left(\frac{\Omega}{2}\right)^{4} \beta_{4}+\left(\frac{\Omega}{2}\right)^{2} \beta_{2}+\beta_{0}=0
$$

The expressions of the coefficients $\beta_{0}, \beta_{2}, \beta_{4}, \beta_{6}, \beta_{8}$ are omitted here due to limed space.

Based on these relationships, the parametric instability analysis can be elaborated. The effects of the considered parameters on the divergence, flutter and parametric instabilities can be investigated. 


\section{Numerical results}

In this section, numerical calculations of the vibration of composite graphene sheets with the small scale, axial speed and viscoelastic foundation effects are performed. The material constants used in these calculations are defined in table 1 .

\begin{tabular}{|l|l|l|l|l|l|}
\hline Material & $\mathrm{E}_{1}(\mathrm{GPa})$ & $\mathrm{E}_{2}(\mathrm{GPa})$ & $\mathrm{G}_{12}$ & $\mathrm{v}_{12}$ & $\rho\left(\mathrm{Kg} / \mathrm{m}^{3}\right)$ \\
\hline $\mathrm{M}_{2}$ & 208 & 18.9 & 5.7 & 0.23 & 2000 \\
\hline
\end{tabular}

Table 1: Material properties of composite graphene sheet

\begin{tabular}{|l|l|l|l|l|l|}
\hline \multirow{2}{*}{ B.C } & \multirow{2}{*}{ method } & \multicolumn{4}{|l|}{ Mode sequence number } \\
\cline { 3 - 6 } & & 1 st & 2nd & 3rt & 4th \\
\hline SSCC & DQM & 4.019 & 4.3865 & 5.098 & 6.064 \\
\cline { 2 - 6 } & FEM [4] & 4.02 & 4.39 & 5.10 & 6.07 \\
\hline \multirow{2}{*}{ SCCC } & DQM & 4.051 & 4.5006 & 5.293 & 6.310 \\
\cline { 2 - 6 } & FEM [4] & 4.05 & 4.50 & 5.29 & 6.31 \\
\hline CCCC & DQM & 4.806 & 5.1025 & 5.704 & 6.573 \\
\cline { 2 - 6 } & FEM [4] & 4.81 & 5.10 & 5.70 & 6.57 \\
\hline
\end{tabular}

Table 2: Frequency parameter for orthotropic plates with various boundary conditions, where $\mu=0, \theta=0, V=0$.

The first fourth dimensionless natural frequencies of orthotropic plates without Winkler-Pasternak visco-elastic foundation and axial peed are calculated by the present analysis and compared with Xing and Liu [4], using finite element method for different boundary conditions are are presented in Table (2). It can be seen; in this comparison study that very good agreements are achieved.

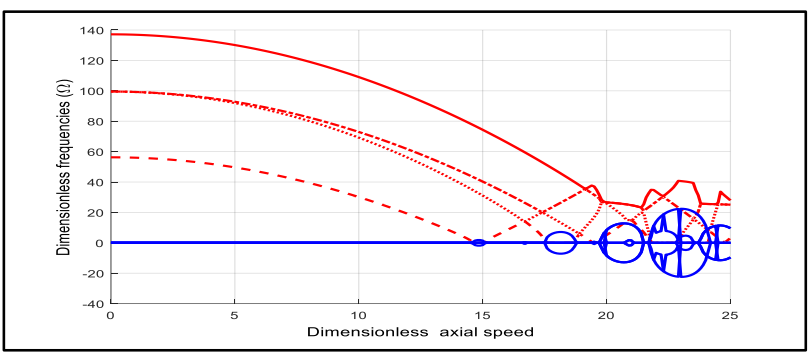

Figure 1. Effect of the axial speed on the divergence and flutter behaviors of a clamped graphene sheet case, with $\mu=0.005, K_{E}=80 ; K_{G}=80 ; \theta=0$.

Figure (1) shows the real and imaginary parts of dimensionless frequencies for clamped boundary conditions. It can be seen that increasing the axial speed, the real part decreases and the imaginary part would reduce, and at axial speed equal to 14.5 the imaginary and real parts of the dimensionless frequency reaches zero for the first mode. This would correspond to a divergence instability for the graphene sheet. At the divergence instability the axial speed is independent of the vibration frequency, since it is a static behavior. When the dimensionless axial speed reaches 18 , the coupled-mode flutter could occur, and at this point, the first and the second modes would combine with each other.

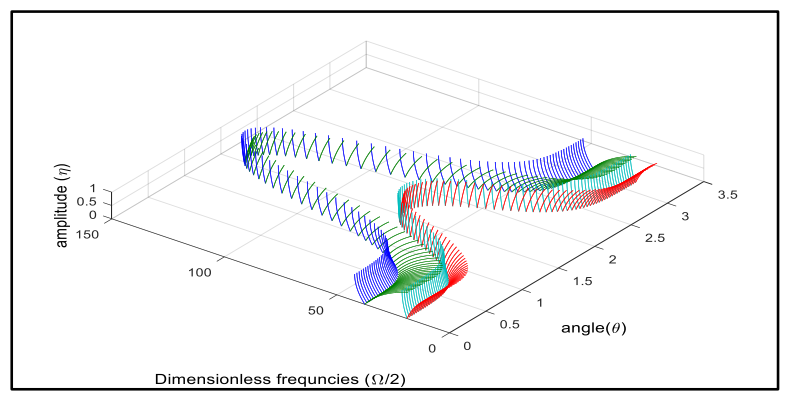

Figure 2. Parametric instability regions of a clamped graphene sheet with respect to the fibers orientation $\theta$ with $\mu=0.005, \mathrm{~V}_{0}=4$.

The evolutions of the parametric instability frequencies and the associated bifurcation points for different fiber angle and a fixed static speed $\mathrm{V}_{0}=4$ in the buckling zone as shown in figure 1 are presented in Figure 2. It can be seen from this figure that with the increase in the fiber angle from 0 to $\pi$ and the dimensionless axial speed the instability zone increases.

\section{Conclusion}

The vibration and instability of nanoplate with visco-elastic foundation are analyzed on basis of the nonlocal elasticity and the Kirchhoff plate theories. The fundamental frequencies and the instability regions are determined and discussed. The effect of the orientation fibers is investigated. Based on the times analysis, it is observed that the free vibration diverges when the parameters $\eta$ and $\Omega$ are chosen in the unstable regions.

\section{Acknowledgements}

The authors would like to acknowledge the financial support of the Deanship of Scientific Research (DSR) at the King Abdulaziz University, Jeddah, Saudi Arabia as well as of the CNRST and the Moroccan Ministry of Higher Education and Scientific Research with the project PPR2/06/2016.

\section{Reference}

[1] M. R. Nami and M. Janghorban, Dynamic analysis of isotropic nanoplates subjected to moving load using statespace method based on nonlocal second order plate theory, Journal of Mechanical Science and Technology, 29, 24232426, 2015.

[2] Azrar A.; Azrar, L.; Aljinaidi, A., Numerical modeling of dynamic and parametric instabilities of single-walled carbon nanotubes conveying pulsating and viscous fluid, Composite Structures Vol. 125, 127-143, 2015.

[3] Azrar A.; Ben Said, M.; Azrar, L.; Aljinaidi, A., Dynamic analysis of Carbon NanoTubes conveying fluid with uncertain parameters and random excitation, Journal Mechanics of Advanced Materials and Structures Vol. 0, No. 0, 1-16, 2018.

[4] Y.F. Xing and B. Liu, New exact solutions for free vibrations of thin orthotropic rectangular plates, Composite structures 89, 567-574, 2009. 